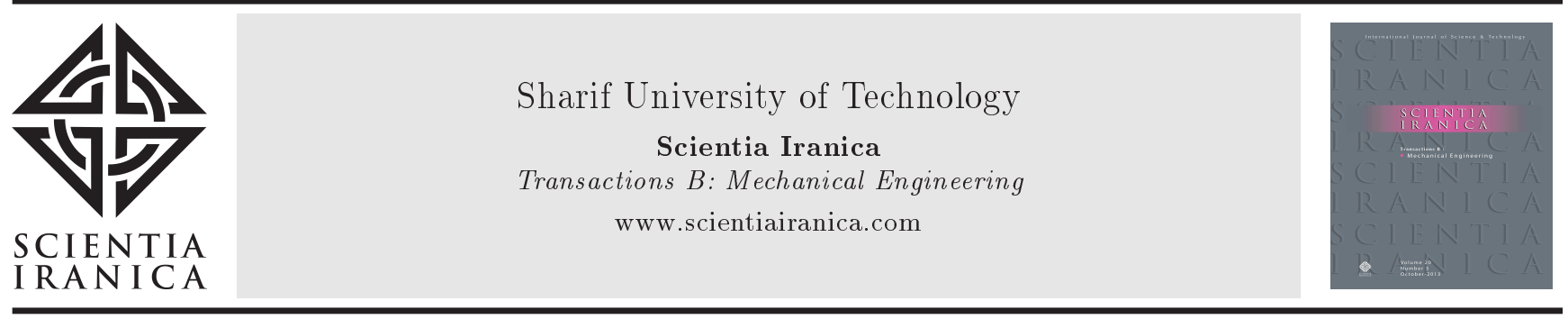

\title{
Effect of size dependency on in-plane vibration of circular micro-rings
}

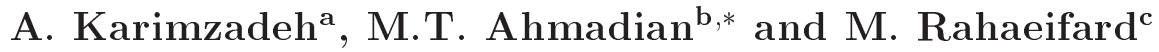 \\ a. School of Mechanical Engineering, Sharif University of Technology, Tehran, Iran. \\ b. Center of Excellence in Design, Robotics and Automation, Sharif University of Technology, Tehran, Iran. \\ c. Department of Mechanical Engineering, Golpayegan University of Technology, Golpayegan, Iran.
}

Received 19 December 2015; accepted 17 April 2017

\section{KEYWORDS}

Micro-ring;

Modified couple stress

theory;

Resonant frequency.

\begin{abstract}
In this paper, based on the modified couple stress theory, the size-dependent dynamic behavior of circular rings on elastic foundation is investigated. The ring is modeled by Euler-Bernoulli and Timoshenko beam theories, and Hamilton's principle is utilized to derive the equations of motion and boundary conditions. The formulation derived is a general form of the equation of motion of circular rings and can be reduced to the classical form by eliminating the size-dependent terms. On this basis, the size-dependent natural frequencies of a circular ring are calculated based on the non-classical Euler-Bernoulli and Timoshenko beam theories. The findings are compared with classical beam theories. Response of the micro-ring under application of static and dynamic loads is investigated and compared with the classical theories. Results show that when the thickness of the ring is in the order of the length scale of the ring material, the natural frequencies evaluated using the modified couple stress are considerably more than those predicted based on the classical beam theories, while the deflection and natural frequencies of the classical and non-classical beam theories approach one another for the rings with thickness much larger than the material length scale.
\end{abstract}

(C) 2017 Sharif University of Technology. All rights reserved.

\section{Introduction}

Circular rings are widely used as micro-gyroscopes in micro-electromechanical systems $[1,2]$. The key block of micro-gyroscopes is a vibrating ring actuated by electrostatic or electromagnetic forces [3,4]. By sensing the lateral vibration of the ring, the angular velocity of the device is calculated. Many researchers have studied the vibration and natural frequencies of circular rings [5,6]. For instance, Chang et al. [5] derived the equations of motion of a circular ring in polar coordi-

*. Corresponding author. Tel: +98 2166165503 ;

Fax: +982166000021

E-mail address: ahmadian@sharif.edu (M.T. Ahmadian) nate using energy method. They used their formulation to calculate the natural frequency and mode shapes of a silicon micro-scale circular ring.

Wu and Parker [6] used perturbation method to derive an analytic expression for natural frequency of circular rings on elastic foundations. Cooley and Parker [7] investigated the vibration behavior of rotating circular rings. They derived the nonlinear equations of motion of the ring using Hamilton's principle and linearized these equations to obtain the natural frequency of the ring. Patel et al. [8] analyzed the vibration of isotropic and orthotropic circular rings. They considered the nonlinearity caused by the large deformation of the ring and used finite-element method to study the mechanical behavior of the ring. 
Rings used in micro-gyroscopes have thicknesses in the order of microns. In the experiments carried out on the micro-scale structures, the size dependency is observed in mechanical behavior of these systems. The response of these components is significantly different from that predicted by the classical theory. Performed experiments revealed that the normalized behavior of micro-mechanical structures is size-dependent, while, according to the classical theory, mechanical response is independent of the structure size. For example, some experiments are conducted to examine the mechanical behavior of micro-wires made of copper [9] and polymeric micro-cantilevers [10]. Outcome of these investigations affirms the size-dependent behavior of these components, and shows that torsional stiffness of micro-wires and bending stiffness of polymeric cantilevers are much more than the stiffness predicted by the classical theory. The size dependency in mechanical behavior of micro structures was also reported by other researchers [11-13].

In contrast to the classical theory which is not able to justify the mechanical response of the micro-scale structures, non-classical theories, such as couple stress theory [14-16] and modified couple stress theory [1719], are appropriately able to explain this phenomenon and cover the gap between the experimental and classical theory results. Couple stress theory as a theory is capable of explaining the size-dependent behavior of mechanisms proposed for the first time by Mindlin and Tiersten [14]. In this theory, new stresses are introduced and considered acting on material element. These stresses were related to higher order derivatives of displacement field with two new constants. The stresses are called higher order stresses, and the new material parameters used in the relation are known as length-scale parameters. A new equilibrium equation, named equilibrium equation of moment of couples, is considered and applied to the relations by Yang et al. [17]. Using this modification, Yang et al. [17] presented the modified couple stress theory.

In recent years, many researchers utilized the modified couple stress theory to analyze the static and dynamic responses of mechanical components such as micro-beams [20-23] and micro-plates [24,25]. The modified couple stress formulations of functionally graded Euler-Bernoulli and Timoshenko beams were developed by Asghari et al. [26,27]. The modified couple stress-based formulation of laminated Timoshenko beams was developed by Chen and $\mathrm{Li}$ [28]. Fakhrabadi et al. [29] analyzed the nonlinear vibration of carbon nanotubes based on modified couple stress theory. Tsiatas [30] utilized the modified couple stress theory to develop the size-dependent formulation of Kirchhoff plates. The formulations of laminated Reddy and Kirchhoff micro plates derived based on the modified couple stress theory were presented by
Chen et al. [31,32]. Static and vibrational analyses of non-classical third-order shear deformation plate were investigated by Gao et al. [33].

The mechanical behavior of fluid conveying microtube was investigated based on the modified couple stress theory by Wang [34]. He derived the natural frequency of the system and calculated the critical flow velocity. On the basis of modified couple stress theory, sensitivity analysis of atomic force microscope was investigated by Kahrobaiyan et al. [35]. The sizedependent static and dynamic behaviors of electrostatically actuated micro-beams were studied by Rahaeifard et al. [36]. They showed that the modified couple stress theory can fill the gap between the experimental and theoretical results of the static pull-in of the microcantilevers.

In this paper, governing equations of motion and boundary conditions of a circular micro-ring on the elastic foundation are derived on the basis of modified couple stress theory and Hamilton's principle. EulerBernoulli and Timoshenko beam theories are considered to obtain two sets of micro-ring formulations. As an example, the size-dependent natural frequencies of circular ring are calculated and compared with the results obtained by the classical theory. Moreover, static and harmonic point forces are applied to the micro-ring and the response is obtained. Findings indicate that for the rings with thickness of the order of the material length-scale parameter, the difference between the non-classical and classical results is notable. Furthermore, for the rings with the radius much more than the thickness, the results of the Timoshenko beam theory converge to those predicted by the EulerBernoulli theory.

\section{Modeling of circular rings}

A simple model of the circular ring on elastic foundation (radial and tangential stiffness) considered in this paper and utilized in Euler-Bernoulli and Timoshenko theory is illustrated in Figure 1.

\subsection{Euler-Bernoulli rings}

The displacement field based on Euler-Bernoulli beam theory in cylindrical coordinate system can be expressed as follows:

$$
\left\{\begin{array}{l}
u_{r}=w \\
u_{\theta}=u+\frac{r}{R} u-\frac{r}{R} \frac{\partial w}{\partial \theta} \\
u_{z}=0
\end{array}\right.
$$

where $w(\theta, t)$ is displacement in radial direction, $u(\theta, t)$ is displacement in tangential direction, $R$ is ring radius, and $r$ denotes the distance of an arbitrary point from the neutral axis of the cross-section of the ring. The infinitesimal strains associated with the displacement 


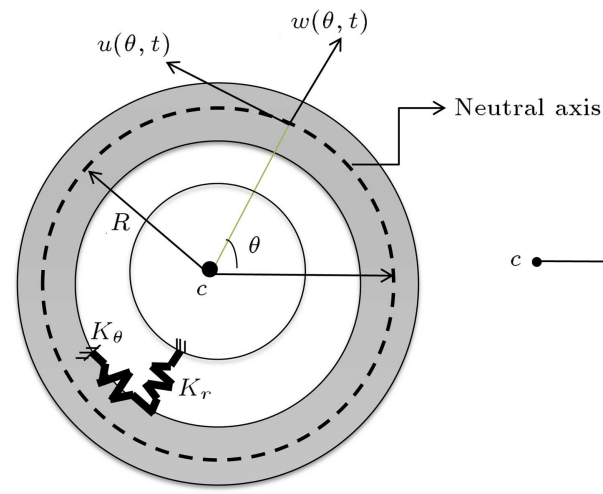

(a)

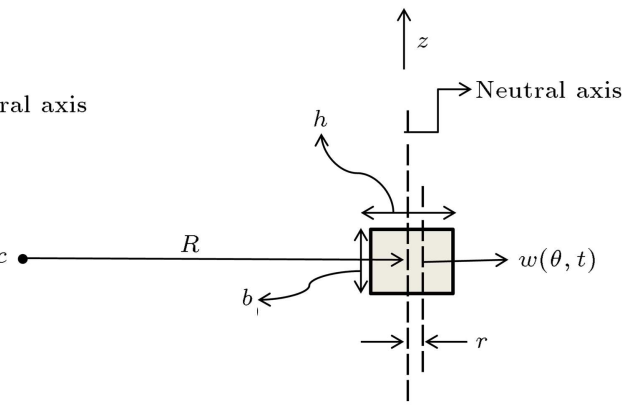

(b)

Figure 1. A circular micro-ring: (a) Geometry and parameters, and (b) cross section of the ring.

field are computed as [37]:

$$
\begin{aligned}
& \varepsilon_{\theta \theta}=\frac{1}{R} \frac{\partial u_{\theta}}{\partial \theta}+\frac{u_{r}}{R}=\frac{1}{R}\left[w+\frac{\partial u}{\partial \theta}+\frac{r}{R}\left(\frac{\partial u}{\partial \theta}-\frac{\partial^{2} w}{\partial \theta^{2}}\right)\right] \\
& \varepsilon_{r r}=\varepsilon_{r \theta}=\varepsilon_{r z}=\varepsilon_{\theta z}=\varepsilon_{z z}=0
\end{aligned}
$$

Moreover, the components of the symmetric curvature tensor can be expressed in terms of the displacement components as [37]:

$$
\begin{aligned}
& \chi_{z \theta}^{s}=\chi_{\theta z}^{s}=\frac{1}{2 R^{2}}\left(\frac{\partial u}{\partial \theta}-\frac{\partial^{2} w}{\partial \theta^{2}}\right), \\
& \chi_{r r}^{s}=\chi_{r \theta}^{s}=\chi_{r z}^{s}=\chi_{\theta \theta}^{s}=\chi_{z z}^{s}=0 .
\end{aligned}
$$

Classical and higher-order stresses $\sigma_{i j}$ and $m_{i j}^{s}$ are also obtained as follows [37]:

$$
\begin{aligned}
& \sigma_{\theta \theta}=\frac{E}{R}\left[w+\frac{\partial u}{\partial \theta}+\frac{r}{R}\left(\frac{\partial u}{\partial \theta}-\frac{\partial^{2} w}{\partial \theta^{2}}\right)\right], \\
& \sigma_{r r}=\sigma_{r \theta}=\sigma_{r z}=\sigma_{\theta z}=\sigma_{z z}=0, \\
& m_{z \theta}^{s}=m_{\theta z}^{s}=\frac{\mu l^{2}}{R^{2}}\left(\frac{\partial u}{\partial \theta}-\frac{\partial^{2} w}{\partial \theta^{2}}\right), \\
& m_{r r}^{s}=m_{r \theta}^{s}=m_{r z}^{s}=m_{\theta \theta}^{s}=m_{z z}^{s}=0 .
\end{aligned}
$$

The variation of potential energy due to a variation in the body configuration can be expressed as [17]:

$$
\begin{aligned}
\delta U= & \iiint_{V}\left(\sigma_{\theta \theta} \delta \varepsilon_{\theta \theta}+2 m_{z \theta} \delta \chi_{z \theta}\right) d V \\
= & \int_{0}^{2 \pi} \iint_{A}\left\{\frac{\sigma_{\theta \theta}}{R}\left(\delta w+\delta \frac{\partial u}{\partial \theta}+\frac{r}{R} \delta \frac{\partial u}{\partial \theta}-\frac{r}{R} \delta \frac{\partial^{2} w}{\partial \theta^{2}}\right)\right. \\
& +2 m_{z \theta}\left(\frac{1}{2 R^{2}}\left(\delta \frac{\partial u}{\partial \theta}-\delta \frac{\partial^{2} w}{\partial \theta^{2}}\right)\right)+K_{r} w \delta w \\
& \left.+K_{\theta} u \delta u\right\} R d A d \theta
\end{aligned}
$$

where $V$ and $A$ represent the volume and cross-section of the micro-ring, and $k_{r}$ and $k_{\theta}$ are radial and tangential stiffness of elastic foundation, respectively. To simplify the derivation of governing equations, stress resultants are defined as follows:

$$
\begin{aligned}
& P=\iint_{A} \sigma_{\theta \theta} d A, \\
& M=\iint_{A} \sigma_{\theta \theta} r d A, \\
& H_{z \theta}=\iint_{A} m_{z \theta} d A,
\end{aligned}
$$

which gives:

$$
\begin{aligned}
\delta U= & \int_{0}^{2 \pi}\left\{\frac{P}{R} \delta w+\frac{P}{R} \delta \frac{\partial u}{\partial \theta}+\frac{M}{R^{2}} \delta \frac{\partial u}{\partial \theta}-\frac{M}{R^{2}} \delta \frac{\partial^{2} w}{\partial \theta^{2}}\right. \\
& \left.+\frac{H_{z \theta}}{R^{2}} \delta \frac{\partial u}{\partial \theta}-\frac{H_{z \theta}}{R^{2}} \delta \frac{\partial^{2} w}{\partial \theta^{2}}+K_{r} w \delta w+K_{\theta} u \delta u\right\} R d \theta
\end{aligned}
$$

Performing some mathematical manipulations yields:

$$
\begin{aligned}
\delta U= & \int_{0}^{2 \pi}\left\{\frac{P}{R} \delta w-\frac{1}{R} \frac{\partial P}{\partial \theta} \delta u-\frac{1}{R^{2}} \frac{\partial M}{\partial \theta} \delta u\right. \\
& -\frac{1}{R^{2}} \frac{\partial^{2} M}{\partial \theta^{2}} \delta w-\frac{1}{R^{2}} \frac{\partial H_{z \theta}}{\partial \theta} \delta u \\
& \left.-\frac{1}{R^{2}} \frac{\partial^{2} H_{z \theta}}{\partial \theta^{2}} \delta w\right\} R d \theta+\left\{\frac{P}{R} \delta u+\frac{M}{R^{2}} \delta u\right. \\
& -\frac{M}{R^{2}} \delta\left(\frac{\partial w}{\partial \theta}\right)+\frac{1}{R^{2}} \frac{\partial M}{\partial \theta} \delta w+\frac{H_{z \theta}}{R^{2}} \delta u \\
& -\frac{H_{z \theta}}{R^{2}} \delta\left(\frac{\partial w}{\partial \theta}\right)+\frac{1}{R^{2}} \frac{\partial H_{z \theta}}{\partial \theta} \delta w+K_{r} w \delta w \\
& \left.+K_{\theta} u \delta u\right\}_{0}^{2 \pi} .
\end{aligned}
$$

Total kinetic energy of the micro-ring is expressed as: 


$$
\begin{gathered}
T=\int_{0}^{2 \pi} \iint_{A} \frac{1}{2} \rho\left[\left(\frac{\partial u_{r}}{\partial t}\right)^{2}+\left(\frac{\partial u_{\theta}}{\partial t}\right)^{2}\right] d V \\
=\int_{0}^{2 \pi} \iint_{A} \frac{1}{2} \rho\left[\left(\frac{\partial w}{\partial t}\right)^{2}+\left(\frac{\partial u}{\partial t}-\frac{r}{R} \frac{\partial^{2} w}{\partial t \partial \theta}\right.\right. \\
\left.\left.+\frac{r}{R} \frac{\partial u}{\partial t}\right)^{2}\right] d V,
\end{gathered}
$$

in which $\rho$ is the density of micro-ring material. Considering the proposed displacement field and ignoring product of small components, the variation of the kinetic energy can be derived as follows:

$$
\partial T=-\rho A \int_{0}^{2 \pi}\left[\frac{\partial^{2} w}{\partial t^{2}} \delta w+\frac{\partial^{2} u}{\partial t^{2}} \delta u\right] R d \theta .
$$

The virtual work done by external forces on the ring is written in the following form:

$$
\delta W=\int_{0}^{2 \pi}[f(\theta, t) \delta w+p(\theta, t) \delta u] R d \theta .
$$

Using Hamilton principle as:

$$
\delta \int(T+W-U) d t=0
$$

gives:

$$
\begin{aligned}
\int_{0}^{T} \int_{0}^{2 \pi}\left\{\left[f R-P+\frac{1}{R} \frac{\partial^{2} M}{\partial \theta^{2}}+\frac{1}{R} \frac{\partial^{2} H_{z \theta}}{\partial \theta^{2}}\right.\right. \\
\left.-\rho A R \frac{\partial^{2} w}{\partial t^{2}}-K_{r} w\right] \delta w+\left[p R+\frac{\partial P}{\partial \theta}\right. \\
+\frac{1}{R} \frac{\partial M}{\partial \theta}+\frac{1}{R} \frac{\partial H_{z \theta}}{\partial \theta}-\rho A R \frac{\partial^{2} u}{\partial t^{2}} \\
\left.\left.-K_{\theta} u\right] \delta u\right\} R d \theta d t+\int_{0}^{T}\left\{\left[-\frac{1}{R} \frac{\partial M}{\partial \theta}\right.\right. \\
\left.-\frac{1}{R} \frac{\partial H_{z \theta}}{\partial \theta}\right] \delta w+\left[-P-\frac{M}{R}-\frac{H_{z \theta}}{R}\right]\left[-\frac{M}{R}\right. \\
\left.\left.-\frac{H_{z \theta}}{R}\right] \delta\left(\frac{\partial w}{\partial \theta}\right)\right\} d t=0 .
\end{aligned}
$$

Performing some straightforward mathematical manipulations gives the governing equations of motion of the Euler-Bernoulli micro-ring as follows:

$$
\begin{aligned}
& f R-P+\frac{1}{R} \frac{\partial^{2} M}{\partial \theta^{2}}+\frac{1}{R} \frac{\partial^{2} H_{z \theta}}{\partial \theta^{2}}-K_{r} w=\rho A R \frac{\partial^{2} w}{\partial t^{2}} \\
& p R+\frac{\partial P}{\partial \theta}+\frac{1}{R} \frac{\partial M}{\partial \theta}+\frac{1}{R} \frac{\partial^{2} H_{z \theta}}{\partial \theta}-K_{\theta} u=\rho A R \frac{\partial^{2} u}{\partial t^{2}} .
\end{aligned}
$$

The corresponding boundary conditions are:

$$
\begin{aligned}
& \frac{1}{R} \frac{\partial M}{\partial \theta}+\frac{1}{R} \frac{\partial H_{z \theta}}{\partial \theta}=0 \quad \text { or } \\
& w=\text { const. } @ \theta=0,2 \pi, \\
& P+\frac{M}{R}+\frac{H_{z \theta}}{R}=0 \quad \text { or } \\
& u=\text { const.@ } \theta=0,2 \pi, \\
& \frac{M}{R}+\frac{H_{z \theta}}{R}=0 \quad \text { or } \\
& \frac{\partial w}{\partial \theta}=\text { const.@ } \theta=0,2 \pi .
\end{aligned}
$$

Considering inextensionality of the centerline of the rings, the following equation should be satisfied [38]:

$$
\varepsilon_{\theta \theta}=0 \text {. }
$$

Satisfying inextentionality condition of the centerline and neglecting product of small quantities in Eqs. (2) gives the following expression for inextensional rings:

$$
w=-\frac{\partial u}{\partial \theta} .
$$

Eqs. (21) and (22) represent the governing equations of Euler-Bernoulli micro-ring. Combining these equations and considering inextensionality of the centerline of the ring, a single differential governing equation is derived as:

$$
\begin{aligned}
\left(1+\frac{\mu A l^{2}}{E I}\right) & \left(\frac{\partial^{6} u}{\partial \theta^{6}}+2 \frac{\partial^{4} u}{\partial \theta^{4}}+\frac{\partial^{2} u}{\partial \theta^{2}}\right)+\frac{R^{4}}{E I}\left(\frac{\partial f}{\partial \theta}+p\right) \\
& +\frac{K_{r} R^{3}}{E I} \frac{\partial^{2} u}{\partial \theta^{2}}-\frac{k_{\theta} R^{3}}{E I} u \\
& +\frac{\rho A R^{4}}{E I} \frac{\partial^{2}}{\partial t^{2}}\left(\frac{\partial^{2} u}{\partial \theta^{2}}-u\right)=0
\end{aligned}
$$

where $I$ is the area moment of inertia defined as $I=$ $\int r^{2} d A$. For convenience, the following dimensionless parameters are introduced:

$$
\begin{aligned}
& \hat{u}=\frac{u}{R} \\
& \hat{f}=\frac{f}{\left(\frac{E I}{R^{3}}\right)}, \quad \hat{p}=\frac{p}{\left(\frac{E I}{R^{3}}\right)}, \\
& \hat{k}_{r}=\frac{K_{r} R^{3}}{E I}, \quad \hat{k}_{\theta}=\frac{K_{\theta} R^{3}}{E I} \\
& \hat{t}=\left(\frac{E I}{\rho A R^{4}}\right)^{\frac{1}{2}} t .
\end{aligned}
$$


Using the introduced dimensionless parameters, the dimensionless governing equation can be written in the following form:

$$
\begin{gathered}
\left(1+\frac{\mu}{E\left(\frac{\bar{r}}{l}\right)^{2}}\right)\left(\frac{\partial^{6} \hat{u}}{\partial \theta^{6}}+2 \frac{\partial^{4} \hat{u}}{\partial \theta^{4}}+\frac{\partial^{2} \hat{u}}{\partial \theta^{2}}\right)+\left(\frac{\partial \hat{f}}{\partial \theta}+\hat{p}\right) \\
+\left(\hat{K}_{r} \frac{\partial^{2} \hat{u}}{\partial \theta^{2}}-\hat{K}_{\theta} \hat{u}\right)+\frac{\partial^{2}}{\partial \hat{t}^{2}}\left(\frac{\partial^{2} \hat{u}}{\partial \theta^{2}}-\hat{u}\right)=0, \quad
\end{gathered}
$$

where $\bar{r}=\sqrt{I / A}$ is radius of gyration of the ring crosssection.

\subsection{Timoshenko rings}

The displacement field of micro-ring with considering the first-order shear deformation in cylindrical coordinate can be stated as follows:

$$
\left\{\begin{array}{l}
u_{r}=w \\
u_{\theta}=u+r \phi \\
u_{z}=0
\end{array}\right.
$$

The infinitesimal strains associated with this displacement field and symmetric curvature tensor can be represented in terms of the displacement variables as follows:

$$
\begin{aligned}
& \varepsilon_{\theta \theta}=\frac{1}{R}\left[w+\frac{\partial u}{\partial \theta}+r \frac{\partial \phi}{\partial \theta}\right], \\
& \varepsilon_{r \theta}=\varepsilon_{\theta r}=\frac{1}{2 R}\left[\frac{\partial w}{\partial \theta}-u+R \phi\right], \\
& \varepsilon_{r r}=\varepsilon_{r z}=\varepsilon_{z r}=\varepsilon_{\theta z}=\varepsilon_{z \theta}=\varepsilon_{z z}=0, \\
& \chi_{z \theta}^{s}=\chi_{\theta z}^{s}=\frac{1}{4 R^{2}}\left(\frac{\partial u}{\partial \theta}-\frac{\partial^{2} w}{\partial \theta^{2}}+R \frac{\partial \phi}{\partial \theta}\right), \\
& \chi_{z r}^{s}=\chi_{r z}^{s}=\frac{1}{4 R^{2}}\left(\frac{\partial w}{\partial \theta}-u+R \phi\right), \\
& \chi_{r r}^{s}=\chi_{r \theta}^{s}=\chi_{\theta r}^{s}=\chi_{\theta \theta}^{s}=\chi_{z z}^{s}=0 .
\end{aligned}
$$

Moreover, the non-zero components of corresponding classical and higher order stresses are obtained as follows:

$$
\begin{aligned}
& \sigma_{\theta \theta}=\frac{E}{R}\left[w+\frac{\partial u}{\partial \theta}+r \frac{\partial \phi}{\partial \theta}\right], \\
& \sigma_{r \theta}=\sigma_{\theta r}=\frac{k \mu}{R}\left[\frac{\partial w}{\partial \theta}-u+R \phi\right], \\
& m_{z \theta}^{s}=m_{\theta z}^{s}=\frac{\mu l^{2}}{2 R^{2}}\left(\frac{\partial u}{\partial \theta}-\frac{\partial^{2} w}{\partial \theta^{2}}+R \frac{\partial \phi}{\partial \theta}\right), \\
& m_{z r}^{s}=m_{r z}^{s}=\frac{\mu l^{2}}{2 R^{2}}\left(\frac{\partial w}{\partial \theta}-u+R \phi\right),
\end{aligned}
$$

where $k$ is numerical factor that depends on the shape of the cross-section of the ring. The variation of strain energy can be calculated as follows:

$$
\begin{aligned}
\delta U= & \iint_{V}\left(\sigma_{\theta \theta} \delta \varepsilon_{\theta \theta}+2 \sigma_{r \theta} \delta \varepsilon_{r \theta}+2 m_{z \theta} \delta \chi_{z \theta}^{s}\right. \\
& \left.+2 m_{z r} \delta \chi_{z r}^{s}\right) d V=\int_{0}^{2 \pi} \iint_{A}\left\{\frac{\sigma_{\theta \theta}}{R}(\delta w\right. \\
& \left.+\delta \frac{\partial u}{\partial \theta}+r \delta \frac{\partial \phi}{\partial \theta}\right)+\frac{\sigma_{r \theta}}{R}\left(\delta \frac{\partial w}{\partial \theta}-\delta u+R \delta \phi\right) \\
& +\frac{m_{z \theta}}{2 R^{2}}\left(\delta \frac{\partial u}{\partial \theta}-\delta \frac{\partial^{2} w}{\partial \theta^{2}}+R \delta \frac{\partial \phi}{\partial \theta}\right)+\frac{m_{z r}}{2 R^{2}}\left(\delta \frac{\delta w}{\delta \theta}\right. \\
& \left.-\delta u+R \delta \phi)+K_{r} w \delta w+K_{\theta} u \delta u\right\} R d A d \theta .
\end{aligned}
$$

In addition to the aforementioned stress resultants, two more stress resultants are defined for Timoshenko rings as follows:

$$
\begin{aligned}
& F=\iint_{A} \sigma_{r \theta} d A, \\
& H_{z r}=\iint_{A} m_{z r}^{s} d A .
\end{aligned}
$$

Then, the variation of strain energy can be rewritten as follows:

$$
\begin{aligned}
\delta U= & \int_{0}^{2 \pi}\left\{\left[\frac{P}{R} \delta w+\frac{P}{R} \delta \frac{\partial u}{\partial \theta}+\frac{M}{R} \delta \frac{\partial \phi}{\partial \theta}\right]+\left[\frac{F}{R} \delta \frac{\partial w}{\partial \theta}-\frac{F}{R} \delta u+F \delta \phi\right]\right. \\
& +\left[\frac{H_{z \theta}}{2 R^{2}} \delta \frac{\partial u}{\partial \theta}-\frac{H_{z} \theta}{2 R^{2}} \delta \frac{\partial^{2} w}{\partial \theta^{2}}+\frac{H_{z} \theta}{2 R} \delta \frac{\partial \phi}{\partial \theta}\right]+\left[K_{r} w \delta w\right. \\
& \left.\left.+K_{\theta} u \delta u\right]+\left[\frac{H_{z r}}{2 R^{2}} \delta \frac{\delta w}{\delta \theta}-\frac{H_{z r}}{2 R^{2}} \delta u+\frac{H_{z r}}{2 R} \delta \phi\right]\right\} R d A d \theta .
\end{aligned}
$$

Using integration by part yields:

$$
\begin{aligned}
\delta U= & \int_{0}^{2 \pi}\left\{\left[\frac{P}{R} \delta w-\frac{1}{R} \frac{\partial P}{\partial \theta} \delta u-\frac{1}{R} \frac{\partial M}{\partial \theta} \delta \phi\right]+\left[-\frac{1}{R} \frac{\partial F}{\partial \theta} \delta w\right.\right. \\
& \left.-\frac{F}{R} \delta u+F D \delta \phi\right]+\left[-\frac{1}{2 R^{2}} \frac{\partial H_{z \theta}}{\partial \theta} \delta u-\frac{1}{2 R^{2}} \frac{\partial^{2} H_{z \theta}}{\partial \theta^{2}} \delta w\right. \\
& \left.-\frac{1}{2 R} \frac{\partial H_{z \theta}}{\partial \theta} \delta \phi\right]+\left[-\frac{1}{2 R^{2}} \frac{\partial H_{z r}}{\partial \theta} \delta w-\frac{H_{z r}}{2 R^{2}} \delta u+\frac{H_{z r}}{2 R} \delta \phi\right] \\
& \left.+\left[K_{r} w \delta w+K_{\theta} u \delta u\right]\right\} R d \theta+R\left\{\left[\frac{P}{R} \delta u+\frac{M}{R} \delta \phi\right.\right. \\
& \left.+\frac{F}{R} \delta w\right]+\left[\frac{H_{z \theta}}{2 R^{2}} \delta u-\frac{H_{z \theta}}{2 R^{2}} \delta \frac{\partial w}{\partial \theta}+\frac{1}{2 R^{2}} \frac{\partial H_{z \theta}}{\partial \theta} \delta w\right. \\
& \left.\left.+\frac{H_{z \theta}}{2 R} \delta \phi\right]+\left[\frac{H_{z r}}{2 R^{2}} \delta w\right]\right\}_{0}^{2 \pi} \cdot
\end{aligned}
$$

Considering the proposed displacement field for Timo- 
shenko ring and ignoring product of small components, the variation of kinetic energy is obtained as follows:

$$
\begin{aligned}
\delta T= & -\int_{0}^{2 \pi}\left[\rho A \frac{\partial^{2} w}{\partial t^{2}} \delta w+\rho A \frac{\partial^{2} u}{\partial t^{2}} \delta u\right. \\
& \left.+\rho I \frac{\partial^{2} \phi}{\partial t^{2}} \delta \phi\right] R d \theta .
\end{aligned}
$$

The variation of external work is written as follows:

$$
\delta W=\int_{0}^{2 \pi}[f(\theta, t) \delta w+p(\theta, t) \delta u] R d \theta .
$$

Using Hamilton's principle gives:

$$
\begin{aligned}
\int_{0}^{T} \int_{0}^{2 \pi}\left\{\left[f R-P+\frac{\partial F}{\partial \theta}+\frac{1}{2 R} \frac{\partial^{2} H_{z \theta}}{\partial \theta^{2}}+\frac{1}{2 R} \frac{\partial H_{z r}}{\partial \theta}\right.\right. \\
\left.-\rho A R \frac{\partial^{2} w}{\partial t^{2}}-K_{r} w\right] \delta w+\left[p R+F+\frac{\partial P}{\partial \theta}\right. \\
\left.+\frac{1}{2 R} \frac{\partial H_{z \theta}}{\partial \theta}+\frac{H_{z r}}{2 R}-\rho A R \frac{\partial^{2} u}{\partial t^{2}}-K_{\theta} u\right] \delta u \\
+\left[\frac{\partial M}{\partial \theta}-F R+\frac{1}{2} \frac{\partial H_{z \theta}}{\partial \theta}-\frac{H_{z r}}{2}\right. \\
\left.\left.-\rho I R \frac{\partial^{2} \phi}{\partial t^{2}}\right] \delta \phi\right\} d \theta d t+\int_{0}^{T}\left\{-\left[P+\frac{H_{z \theta}}{2 R}\right] \delta u\right. \\
-\left[F+\frac{1}{2 R} \frac{\partial H_{z \theta}}{\partial \theta}+\frac{H_{z r}}{2 R}\right] \delta w-\left[M+\frac{H_{z \theta}}{2}\right] \delta \phi \\
\left.\left.+\frac{H_{z \theta}}{2 R} \delta\left(\frac{\partial u}{\partial \theta}\right)\right\} d t_{0}^{2 \pi}\right\}=0 .
\end{aligned}
$$

The equation of motion for the Timoshenko circular ring and the corresponding boundary conditions are derived as follows:

$$
\begin{gathered}
f R-P+\frac{\partial F}{\partial \theta}+\frac{1}{2 R} \frac{\partial^{2} H_{z \theta}}{\partial \theta^{2}}+\frac{1}{2 R} \frac{\partial H_{z \theta}}{\partial \theta}-K_{r} w \\
=\rho A R \frac{\partial^{2} w}{\partial t^{2}} \\
p R+F+\frac{\partial P}{\partial \theta}+\frac{1}{2 R} \frac{\partial H_{z \theta}}{\partial \theta}+\frac{H_{z r}}{2 R}-K_{\theta} u \\
=\rho A R \frac{\partial^{2} u}{\partial t^{2}}, \\
\frac{\partial M}{\partial \theta}-F R+\frac{1}{2} \frac{\partial H_{z \theta}}{\partial \theta}-\frac{H_{z r}}{2}=\rho I R \frac{\partial^{2} \phi}{\partial t^{2}}, \\
P+\frac{H_{z \theta}}{2 R}=0 \\
u=\text { const. } @ \theta=0,2 \pi,
\end{gathered}
$$

$$
\begin{aligned}
& F+\frac{1}{2 R} \frac{\partial H_{z \theta}}{\partial \theta}+\frac{H_{z r}}{2 R}=0 \quad \text { or } \\
& w=\text { const.@ } \theta=0,2 \pi, \\
& M+\frac{H_{z \theta}}{2}=0 \quad \text { or } \\
& \phi=\text { const.@ } \theta=0,2 \pi,
\end{aligned}
$$

$$
\frac{H_{z \theta}}{2 R}=0 \quad \text { or }
$$$$
\frac{\partial w}{\partial \theta}=\text { const. } @ \theta=0,2 \pi
$$

Using the governing equations of Timoshenko ring and considering inextensionality of the centerline of the ring, a single differential governing equation for the Timoshenko micro-ring is derived as follows:

$$
\begin{aligned}
\frac{\partial^{8} u}{\partial \theta^{8}}\left[-\frac{l^{2}}{4 k R^{2}}\right]+\frac{\partial^{6} u}{\partial \theta^{6}}\left[\frac{\mu A l^{2}}{E I}\left(1+\frac{l^{2}}{4 k R^{2}}\right)\right. \\
\left.+\left(1-\frac{l^{2}}{4 k R^{2}}\right)\right]+\frac{\partial^{4} u}{\partial \theta^{4}}\left[\frac{2 \mu A l^{2}}{E I}\left(1+\frac{l^{2}}{4 k R^{2}}\right)\right. \\
\left.+\left(2+\frac{l^{2}}{4 k R^{2}}\right)\right]+\frac{\partial^{2} u}{\partial \theta^{2}}\left[\left(\frac{\mu A l^{2}}{E I}+1\right)\right. \\
\left.\left.+\frac{l^{2}}{4 k R^{2}}\right)\right]+\frac{\partial^{8} u}{\partial \theta^{6} \partial t^{2}}\left[\frac{\rho l^{2}}{4 k E}\right] \\
+\frac{\partial^{6} u}{\partial \theta^{4} \partial t^{2}}\left[-\frac{\rho R^{2}}{E}\left(1-\frac{l^{2}}{4 k R^{2}}+\frac{A l^{2}}{4 k I}\right)\right. \\
\left.+\frac{\rho R^{2}}{k \mu}\right]+\frac{\partial^{4} u}{\partial \theta^{2} \partial t^{2}}\left[-\frac{\rho A R^{4}}{E I}\left(1+\frac{l^{2}}{2 k R^{2}}\right.\right. \\
+\frac{K_{r} R^{3}}{E I} \frac{\partial^{2} u}{\partial \theta^{2}}-\frac{K_{\theta} R^{3}}{E I} u+\frac{R^{4}}{E I}\left(1+\frac{l^{2}}{4 k R^{2}}\right) \\
+\frac{\partial^{6} u}{\partial \theta^{2} \partial t^{4}}\left[\frac{\rho^{2} R^{4}}{k E \mu}\right]-\frac{\partial R^{4} u}{\partial t^{4}}\left[\frac{\rho^{2} R^{4}}{k E \mu}\right] \\
\left.+\frac{\partial^{2} u}{\partial t^{2}}\left[\left(\frac{\rho A R^{4}}{E I}\right)+\frac{\rho R^{2}}{E}\right)\left(1+\frac{l^{2}}{4 k R^{2}}\right)\right]
\end{aligned}
$$


Employing introduced dimensionless parameters yields:

$$
\begin{aligned}
& \frac{\partial^{8} \hat{u}}{\partial \theta^{8}}\left[-\frac{1}{4 k\left(\frac{R}{l}\right)^{2}}\right]+\frac{\partial^{6} \hat{u}}{\partial \theta^{6}}\left[\frac{\mu}{E\left(\frac{\bar{r}}{l}\right)^{2}}\left(1+\frac{1}{4 k\left(\frac{R}{l}\right)^{2}}\right)\right. \\
& \left.+\left(1-\frac{1}{4 k\left(\frac{R}{l}\right)^{2}}\right)\right]+\frac{\partial^{4} \hat{u}}{\partial \theta^{4}}\left[\frac{2 \mu}{E\left(\frac{\bar{r}}{l}\right)^{2}}\left(1+\frac{1}{4 k\left(\frac{R}{l}\right)^{2}}\right)\right. \\
& \left.+\left(2+\frac{1}{4 k\left(\frac{R}{l}\right)^{2}}\right)\right]+\frac{\partial^{2} \hat{u}}{\partial \theta^{2}}\left[\left(\frac{\mu}{E\left(\frac{\bar{r}}{l}\right)^{2}}+1\right)\right. \\
& \left.\left(1+\frac{1}{4 k\left(\frac{R}{l}\right)^{2}}\right)\right]+\frac{\partial^{8} \hat{u}}{\partial \theta^{6} \partial \hat{t}^{2}}\left[\frac{1}{4 k\left(\frac{R}{l}\right)^{2}\left(\frac{R}{\bar{r}}\right)^{2}}\right] \\
& +\frac{\partial^{6} \hat{u}}{\partial \theta^{4} \partial \hat{t}^{2}}\left[-\frac{1}{\left(\frac{R}{\bar{r}}\right)^{2}}\left(1-\frac{1}{4 k\left(\frac{R}{l}\right)^{2}}\right.\right. \\
& \left.\left.+\frac{1}{4 k\left(\frac{\bar{r}}{l}\right)^{2}}+\frac{E}{k \mu}\right)\right]+\frac{\partial^{4} \hat{u}}{\partial \theta^{2} \partial \hat{t}^{2}}[(1 \\
& \left.+\frac{1}{2 k\left(\frac{R}{l}\right)^{2}}+\frac{E}{k \mu\left(\frac{R}{\bar{r}}\right)^{2}}\right) \\
& \left.-\frac{1}{\left(\frac{R}{\bar{r}}\right)^{2}}\left(2+\frac{1}{4 k\left(\frac{R}{l}\right)^{2}}\right)\right]+\left(\hat{K}_{r} \frac{\partial^{2} \hat{u}}{\partial \theta^{2}}-\hat{K}_{\theta} \hat{u}\right) \\
& +\frac{\partial^{6} \hat{u}}{\partial \theta^{2} \partial \hat{t}^{4}}\left[\frac{E}{k \mu\left(\frac{R}{\bar{r}}\right)^{4}}\right]-\frac{\partial^{4} \hat{u}}{\partial \hat{t}^{4}}\left[\frac{E}{k \mu\left(\frac{R}{\bar{r}}\right)^{4}}\right] \\
& -\frac{\partial^{2} \hat{u}}{\partial \hat{t}^{2}}\left[\left(1+\frac{1}{\left(\frac{R}{\bar{r}}\right)^{2}}\right)\left(1+\frac{1}{4 k\left(\frac{R}{l}\right)^{2}}\right)\right] \\
& +\left(1+\frac{1}{4 k\left(\frac{R}{l}\right)^{2}}\right)\left(\hat{p}+\frac{\partial \hat{f}}{\partial \theta}\right)-\left(\frac{1}{4 k\left(\frac{R}{l}\right)^{2}}\right. \\
& \left.+\frac{E}{k \mu\left(\frac{R}{\bar{r}}\right)^{2}}\right) \frac{\partial^{2}}{\partial \theta^{2}}\left(\hat{p}+\frac{\partial \hat{f}}{\partial \theta}\right) \\
& +\frac{E}{k \mu\left(\frac{R}{\bar{r}}\right)^{4}} \frac{\partial^{2}}{\partial \hat{t}^{2}}\left(\hat{p}+\frac{\partial \hat{f}}{\partial \theta}\right)=0 .
\end{aligned}
$$

\section{Static case}

In this section, the response of micro-ring under static load is investigated. For this reason, as a case study, external point loads are exerted on the ring diagonally in orientation $\pi / 3$ from the horizontal direction as shown in Figure 2. In the static load case, the timedependent derivatives are ignored and the radial force acting on the ring is considered to be:

$$
\begin{aligned}
& \hat{f}(\theta)=F_{0} \delta\left(\theta-\frac{\pi}{3}\right)+F_{0} \delta\left(\theta-\frac{4 \pi}{3}\right), \\
& \hat{p}(\theta)=0,
\end{aligned}
$$

where $F_{0}$ is amplitude of static load, and $\pi / 3$ and $4 \pi / 3$ express the positions of the forces. The governing equation of Euler-Bernoulli micro-ring under static load would be as follows:

$$
\begin{aligned}
(1+ & \left.\frac{\mu}{E\left(\frac{\bar{r}}{l}\right)^{2}}\right)\left(\frac{\partial^{6} \hat{u}}{\partial \theta^{6}}+2 \frac{\partial^{4} \hat{u}}{\partial \theta^{4}}+\frac{\partial^{2} \hat{u}}{\partial \theta^{2}}\right) \\
+ & F_{0} \frac{\partial}{\partial \theta}\left(\delta\left(\theta-\frac{\pi}{3}\right)+\delta\left(\theta-\frac{4 \pi}{3}\right)\right) \\
& +\left(\hat{K}_{r} \frac{\partial^{2} \hat{u}}{\partial \theta^{2}}-\hat{K}_{\theta} \hat{u}\right)=0 .
\end{aligned}
$$

In a similar procedure, the governing equation of Timoshenko ring for static loading can be written as follows:

$$
\begin{aligned}
\frac{\partial^{8} \hat{u}}{\partial \theta^{8}}[ & \left.-\frac{1}{4 k\left(\frac{R}{l}\right)^{2}}\right]+\frac{\partial^{6} \hat{u}}{\partial \theta^{6}}\left[\frac{\mu}{E\left(\frac{\bar{r}}{l}\right)^{2}}\left(1+\frac{1}{4 k\left(\frac{R}{l}\right)^{2}}\right)\right. \\
& \left.+\left(1-\frac{1}{4 k\left(\frac{R}{l}\right)^{2}}\right)\right]+\frac{\partial^{4} \hat{u}}{\partial \theta^{4}}\left[\frac{2 \mu}{E\left(\frac{\bar{r}}{l}\right)^{2}}\left(1+\frac{1}{4 k\left(\frac{R}{l}\right)^{2}}\right)\right. \\
& \left.+\left(2+\frac{1}{4 k\left(\frac{R}{l}\right)^{2}}\right)\right] \\
& +\frac{\partial^{2} \hat{u}}{\partial \theta^{2}}\left[\left(\frac{\mu}{E\left(\frac{\bar{r}}{l}\right)^{2}}+1\right)\left(1+\frac{1}{4 k\left(\frac{R}{l}\right)^{2}}\right)\right] \\
& +\left(\hat{K}_{r} \frac{\partial^{2} \hat{u}}{\partial \theta^{2}}-\hat{K}_{\theta} \hat{u}\right)+\left(1+\frac{1}{4 k\left(\frac{R}{l}\right)^{2}}\right) \\
& F_{0} \frac{\partial}{\partial \theta}\left(\delta\left(\theta-\frac{\pi}{3}\right)+\delta\left(\theta-\frac{4 \pi}{3}\right)\right) \\
& -\left(\frac{1}{4 k\left(\frac{R}{l}\right)^{2}}+\frac{E}{k \mu\left(\frac{R}{\bar{r}}\right)^{2}}\right) F_{0} \frac{\partial^{3}}{\partial \theta^{3}}\left(\delta\left(\theta-\frac{\pi}{3}\right)\right. \\
& \left.+\delta\left(\theta-\frac{4 \pi}{3}\right)\right)=0 .
\end{aligned}
$$

The response of a structure under application of external forces can be obtained using mode summation technique. Based on this method, the solution is expressed as the summation of the mode shapes of the structure. Therefore, the mode shapes of the ring are 


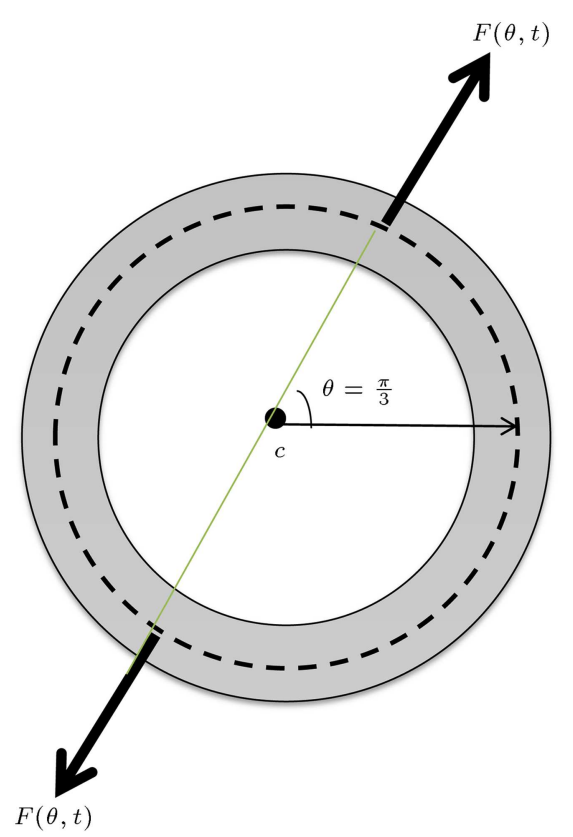

Figure 2. Circular micro-ring loading.

needed. This mode which is satisfying the governing equations and boundary conditions can be mentioned as follows:

$$
U_{n}=\sin (n \theta+\phi) \quad n=2,3, \ldots,
$$

where $n$ is the mode number of the ring, noting that $n=1$ indicates the rigid body motion of the ring. Using the mode shapes, the response of the micro ring under static loading can be expressed as follows:

$$
\hat{u}(\theta)=\sum_{n=2}^{\infty} C_{n} \sin (n \theta+\phi) .
$$

By inserting the above equation into the governing equations of Euler-Bernoulli and Timoshenko ring with sufficient modes and using Galerkin approximation, one can obtain the response of the ring.

\section{Free vibration analysis}

\subsection{Natural frequency of Euler-Bernoulli ring}

To find natural frequencies of the ring, external forces are assumed to be zero and harmonic response is considered as follows:

$$
\hat{u}(\theta, \hat{t})=U(\theta) e^{i \omega \hat{t}},
$$

where $\omega$ is dimensionless natural frequency of the micro-ring. Eq. (59) can be rewritten as follows:

$$
\begin{aligned}
(1 & \left.+\frac{\mu}{E\left(\frac{\bar{r}}{l}\right)^{2}}\right)\left(\frac{\partial^{6} U}{\partial \theta^{6}}+2 \frac{\partial^{4} U}{\partial \theta^{4}}+\frac{\partial^{2} U}{\partial \theta^{2}}\right) \\
& +\left(\hat{K}_{r} \frac{\partial^{2} U}{\partial \theta^{2}}-\hat{K}_{\theta} U\right)-\omega^{2}\left(\frac{\partial^{2} U}{\partial \theta^{2}}-U\right)=0 .
\end{aligned}
$$

As it is expressed in the static case section, the solution of this equation can be proposed in the following form:

$$
U(\theta)=c_{0} \sin (n \theta+\phi) \quad n=2,3, \ldots,
$$

in which $c_{0}$ is a real constant. The natural frequency of Euler-Bernoulli micro-ring, according to the modified couple stress theory, is derived as follows:

$$
\omega^{2}=\left(1+\frac{\mu}{E\left(\frac{\bar{r}}{l}\right)^{2}}\right)\left(\frac{n^{6}-2 n^{4}+n^{2}}{n^{2}+1}\right)+\left(\frac{\hat{K}_{r} n^{2}+\hat{K}_{\theta}}{n^{2}+1}\right) .
$$

\subsection{Natural frequency of Timoshenko ring}

As performed in the previous section, external forces are assumed to be equal to zero and the following harmonic response is considered for the ring:

$$
\hat{u}(\theta, \hat{t})=c_{0} \sin (n \theta+\phi) e^{i \omega \hat{t}} \quad n=2,3, \ldots .
$$

Utilizing the proposed relation, the following algebraic equation is derived for natural frequency of the Timoshenko micro-ring:

$$
A \omega^{4}-B \omega^{2}+C=0,
$$

in which:

$$
\begin{aligned}
& A=\left[\left(n^{2}+1\right)\left(\frac{E}{k \mu\left(\frac{R}{\bar{r}}\right)^{4}}\right)\right], \\
& B=\left[n^{6}\left(\frac{1}{4 k\left(\frac{R}{l}\right)^{2}\left(\frac{R}{\bar{r}}\right)^{2}}\right)+n^{4}\left(\frac { 1 } { ( \frac { R } { \overline { r } } ) ^ { 2 } } \left(1-\frac{1}{4 k\left(\frac{R}{l}\right)^{2}}\right.\right.\right. \\
& \left.\left.+\frac{1}{4 k\left(\frac{\bar{r}}{l}\right)^{2}}+\frac{E}{k \mu}\right)\right) \\
& +n^{2}\left(\left(1+\frac{1}{2 k\left(\frac{R}{l}\right)^{2}}+\frac{E}{k \mu\left(\frac{R}{\bar{r}}\right)^{2}}\right)\right. \\
& \left.-\frac{1}{\left(\frac{R}{\bar{r}}\right)^{2}}\left(2+\frac{1}{4 k\left(\frac{R}{l}\right)^{2}}\right)\right)+\left(1+\frac{1}{\left(\frac{R}{\bar{r}}\right)^{2}}\right)(1 \\
& \left.\left.+\frac{1}{4 k\left(\frac{R}{l}\right)^{2}}\right)\right] \\
& C=\left[n^{8}\left(\frac{1}{4 k\left(\frac{R}{l}\right)^{2}}\right)+n^{6}\left(\frac{\mu}{E\left(\left(\frac{\bar{r}}{l}\right)^{2}\right)}\left(1+\frac{1}{4 k\left(\frac{R}{l}\right)^{2}}\right)\right.\right. \\
& \left.+\left(1-\frac{1}{4 k\left(\frac{R}{l}\right)^{2}}\right)\right)+n^{4}\left(\frac{2 \mu}{E\left(\frac{\bar{r}}{l}\right)^{2}}(1\right. \\
& \left.\left.+\frac{1}{4 k\left(\frac{R}{l}\right)^{2}}\right)+\left(2+\frac{1}{4 k\left(\frac{R}{l}\right)^{2}}\right)\right) \\
& \left.+n^{2}\left(\left(1+\frac{\mu}{E\left(\frac{\bar{r}}{l}\right)^{2}}\right)\left(1+\frac{1}{4 k\left(\frac{R}{l}\right)^{2}}\right)\right)\right] \\
& +\left(\hat{K}_{r} n^{2}+\hat{K}_{\theta}\right) .
\end{aligned}
$$




\section{Harmonic case}

In the case of harmonic load, the radial force acting on the ring is considered to have the form as follows:

$$
\begin{aligned}
f(\theta, t)= & F_{0} \sin (\Omega t) \delta\left(\theta-\frac{\pi}{3}\right) \\
& +F_{0} \sin (\Omega t) \delta\left(\theta-\frac{4 \pi}{3}\right),
\end{aligned}
$$

where $\Omega$ is the excitation frequency. The governing equation of Euler-Bernoulli micro-ring under harmonic loading would be as follows:

$$
\begin{aligned}
(1 & \left.+\frac{\mu}{E\left(\frac{\bar{r}}{l}\right)^{2}}\right)\left(\frac{\partial^{6} \hat{u}}{\partial \theta^{6}}+2 \frac{\partial^{4} \hat{u}}{\partial \theta^{4}}+\frac{\partial^{2} \hat{u}}{\partial \theta^{2}}\right) \\
& +F_{0} \sin (\Omega t) \frac{\partial}{\partial \theta}\left(\delta\left(\theta-\frac{\pi}{3}\right)+\delta\left(\theta-\frac{4 \pi}{3}\right)\right) \\
& +\left(\hat{K}_{r} \frac{\partial^{2} \hat{u}}{\partial \theta^{2}}-\hat{K}_{\theta} \hat{u}\right)+\frac{\partial^{2}}{\partial \hat{t}^{2}}\left(\frac{\partial^{2} \hat{u}}{\partial \theta^{2}}-\hat{u}\right)=0 .
\end{aligned}
$$

Utilizing the mode summation method, the deflection of the ring can be considered as follows:

$$
U(\theta, t)=\sum_{n=2}^{\infty} \eta(t) \sin (n \theta+\phi) .
$$

Inserting the above equation into Eq. (78) gives the following differential equation for $\eta(t)$ which can be easily solved:

$$
\begin{gathered}
\left(n^{2}+1\right) \frac{\partial^{2} \eta(t)}{\partial t^{2}}+\left(1+\frac{\mu}{E\left(\frac{\bar{r}}{l}\right)^{2}}\right)\left(n^{6}-2 n^{4}\right. \\
\left.+n^{2}\right) \eta(t)+\left(\hat{K}_{r} n^{2}+\hat{K}_{\theta}\right) \eta(t)=N(t)
\end{gathered}
$$

where:

$$
\begin{aligned}
N(t)= & \frac{1}{\pi} \int_{0}^{2 p}\left(F_{0} \sin (\Omega t) \frac{\partial}{\partial \theta}\left(\delta\left(\theta-\frac{\pi}{3}\right)\right)\right. \\
& \left.+F_{0} \sin (\Omega t) \frac{\partial}{\partial \theta}\left(\delta\left(\theta-\frac{4 \pi}{3}\right)\right)\right) \sin (n \theta+\phi) d \theta
\end{aligned}
$$

After the time part of deflection $(\eta(t))$ is solved, the response of the Euler-Bernoulli micro ring can be obtained from the mode summation technique by multiplying the time part solution to corresponding mode. A similar procedure is performed on Timoshenko ring and the differential equation for time part of response is derived as follows:

$$
A \frac{\partial^{4} \eta(t)}{\partial t^{4}}+B \frac{\partial^{2} \eta(t)}{\partial t^{2}}+C \eta(t)=N(t)
$$

where $A, B$, and $C$ coefficients are the same as Eqs. (74) to (76), and $N(t)$ can be achieved from Eq. (81). By solving the time part solution of Timoshenko ring and multiplying them to the corresponding modes, the response of Timoshenko ring can be obtained.

\section{Results and discussion}

Ring with rectangular cross-section is considered as a case study in this section. Let parameters $h$ and $b$ denote the height and width of the ring cross-section, respectively. These parameters' radius of gyration of the cross-section of the ring may be replaced by $(1 / 2 \sqrt{3}) h$.

The first dimensionless natural frequency of Euler-Bernoulli micro-ring with respect to the ratio of ring thickness to the material length scale is illustrated in Figure 3. The dimensionless coefficients of elastic foundation $\left(\hat{K}_{r}, \hat{K}_{\theta}\right)$ are assumed to be zero. The size dependency of the normalized natural frequency of the Euler-Bernoulli micro-ring can be seen clearly in this figure. It can be easily concluded that when the ratio of $h / l$ is less than 10 , the difference between the natural frequencies given by the modified couple stress and classical theories is considerable. As is well-known in the literature and can be understood from this figure, the modified couple stress theory predicts the stiffness of the micro-scale structures higher than the classical theory. Hence, the natural frequency predicted by the modified couple stress theory is higher than that evaluated by that classical theory.

The first dimensionless natural frequencies of Timoshenko ring are plotted in Figure 4 . The dimensionless coefficients of elastic foundation $\left(\hat{K}_{r}, \hat{K}_{\theta}\right)$ are assumed to be zero. To calculate the results presented in this figure, the ratio of the ring radius to the thickness of the cross-section is considered as $R / h=10$. The results indicate that when the thickness of the cross-section is in the order of the material length scale, the difference between classical and non-classical theories is considerable. As an example for $h / l=3$, this difference is $25 \%$. This difference means that size effect should be considered for the structures in which the constitutive length (e.g., the thickness of the beam-

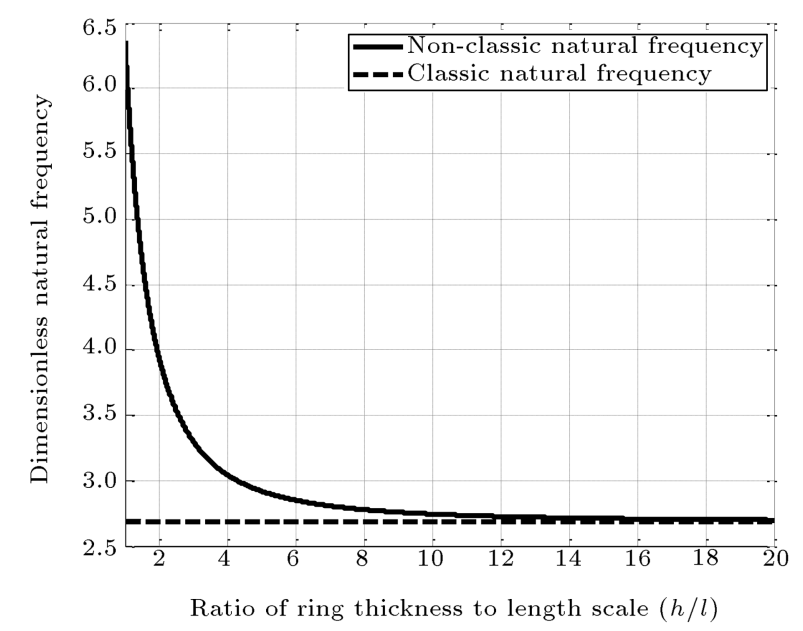

Figure 3. First dimensionless natural frequency of Euler-Bernoulli ring. 


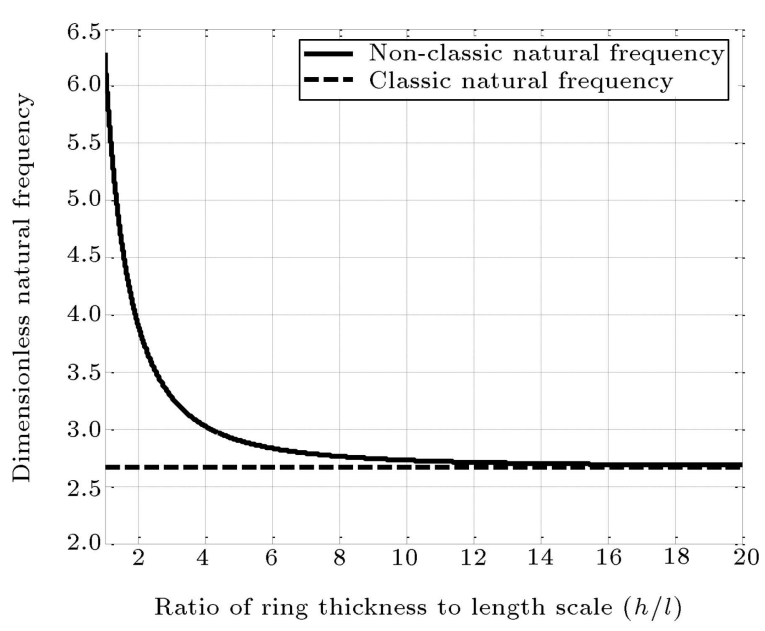

Figure 4. First dimensionless natural frequency of Timoshenko ring.

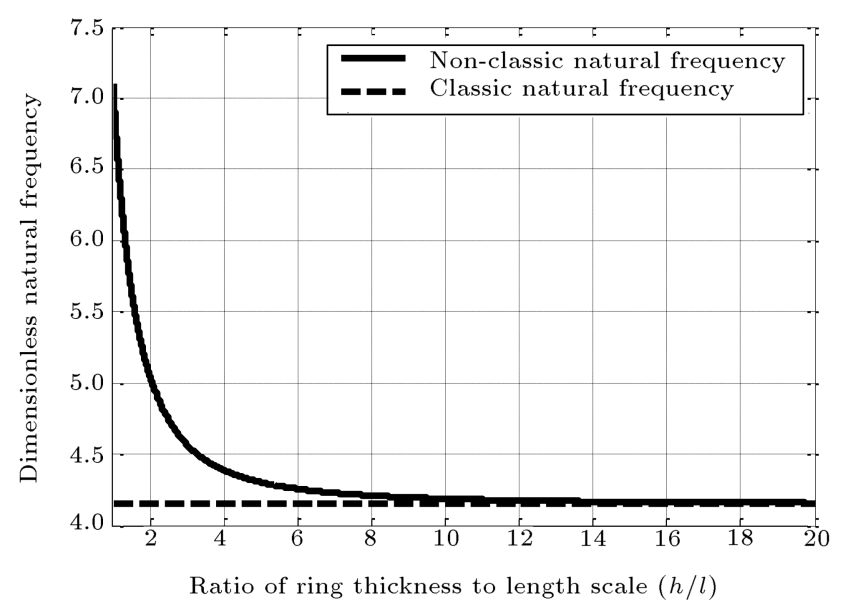

Figure 5. First dimensionless natural frequency of Euler-Bernoulli ring on elastic foundation.

type structure) is in the order of material length-scale parameter.

In Figure 5, the first dimensionless natural frequencies of Euler-Bernoulli micro-ring in terms of stiffness coefficient are presented. Results indicated that natural frequency increases as radial and tangential stiffness coefficient enhances. The same result can be obtained from Figure 6 in which the first dimensionless natural frequencies of Timoshenko micro-ring in terms of stiffness coefficient are investigated. The dimensionless coefficients of elastic foundation are assumed to be $\widehat{K}_{r}=\widehat{K}_{\theta}=10$.

The ratios of the 1 st, 5 th, and 10 th natural frequencies of Timoshenko ring to those of the EulerBernoulli are presented in Figure 7 for $h / l=3$. Based on this figure, it can be realized that the difference between the natural frequencies given by the Timoshenko and Euler-Bernoulli theories would be larger as the number of the mode increases. Furthermore, as the ratio of ring radius to ring thickness increases

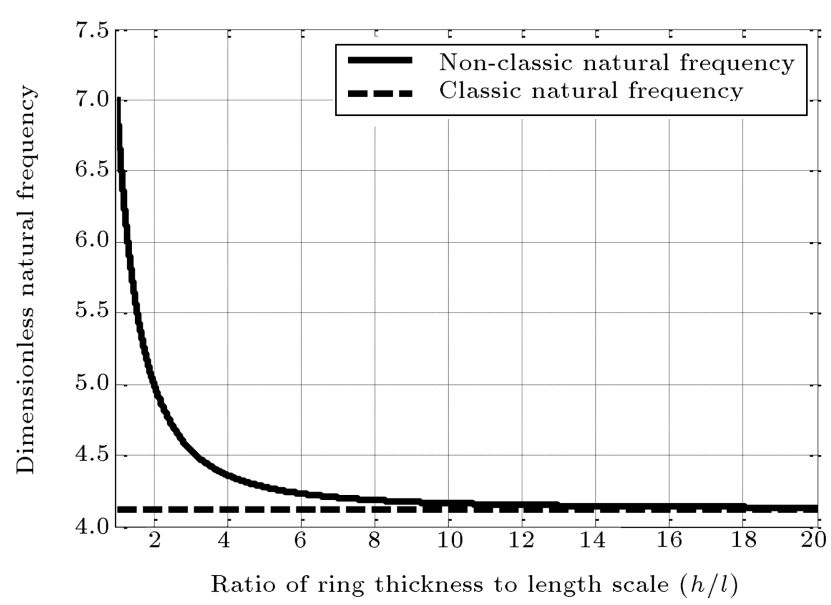

Figure 6. First dimensionless natural frequency of Timoshenko ring on elastic foundation.

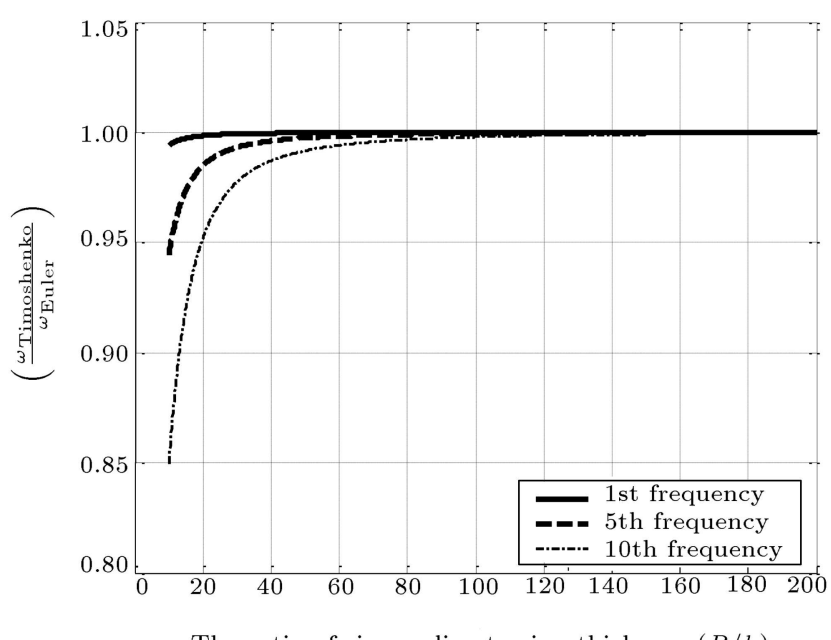

Figure 7. Natural frequency of Timoshenko ring over that of Euler ring for first, fifth and tenth frequencies.

the difference between the natural frequencies of the Timoshenko ring and that of the Euler-Bernoulli ring tend to each other. However, for the values larger than 10, the natural frequencies of micro-rings for Timoshenko and Euler beams are assumed to be the same.

Figure 8 shows the effect of stiffness coefficient of elastic foundation on size-dependent mechanical behavior of micro-ring. In this figure, the ratio of size-dependent natural frequency to classic natural frequency with respect to radial and tangential stiffness coefficients is plotted for $R / h>20$. This figure reveals that as the stiffness coefficient of the system increases, the size-dependent parameter would be less effective in natural frequency of the system, and the ratio of nonclassic to classic natural frequencies tends to 1 .

Utilizing the mode summation technique and the derived equations, the response of the micro ring under static load for $R / h>20$ and $h / l=3$ is presented in 


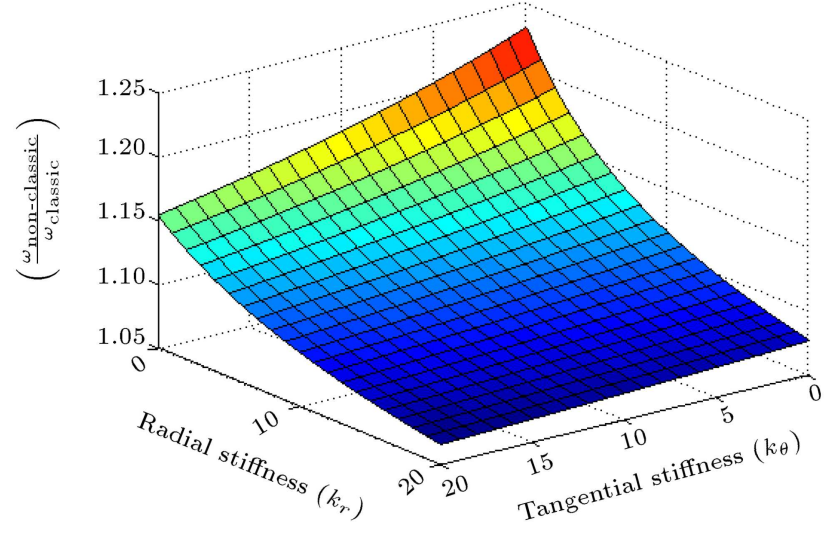

Figure 8. Ratio of non-classic to classic natural frequency with respect to elastic foundation stiffness.

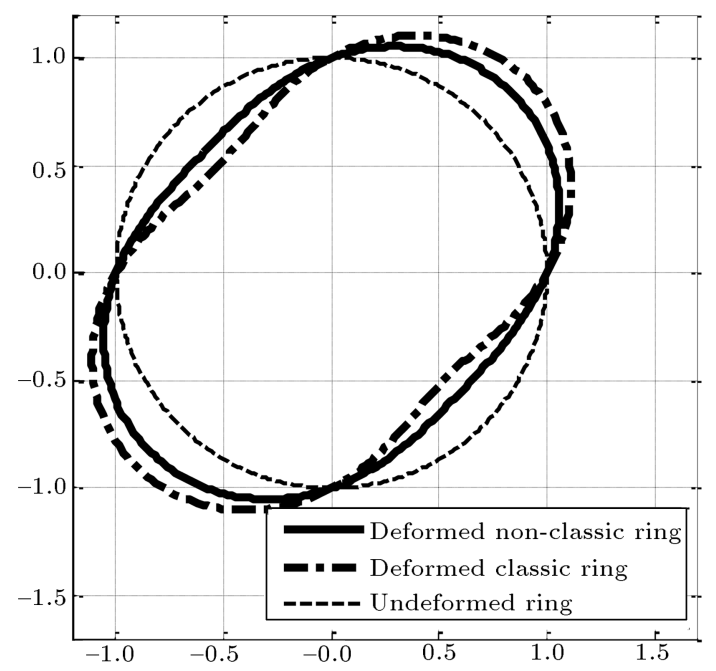

Figure 9. Maximum deflection of micro-ring under static load $h / l=3$.

Figure 9. It should be noted that since the ratio of ring radius to ring thickness $(R / h)$ is assumed to be higher than 20, the results of static loading based on Euler-Bernoulli and Timoshenko theories match each other. As it is expected from the free vibration analysis, micro ring based on non-classical theory is stiffer than classical theory and deflection is smaller compared to classical theory.

Using mode summation technique and considering 4 modes of the ring, the effect of size dependency on the maximum deflection of the micro ring at different ratios of $h / l$ under harmonic loading is plotted in Figure 10 . The excitation frequency $(\Omega)$ is assumed to be close to the first natural frequency of the ring. Findings indicate that as $h / l$ increases, the classical and nonclassical results tend to one another (for $h / l=3$, there is a meaningful gap between classic and non-classic responses, while the responses completely match each other $h / l>100)$. Comparing the natural frequencies and deflection of the micro ring under external force based on classical and non-classical theories reveals that for the vibrating ring gyroscope which operates based on natural frequency of the micro ring, the size dependency must be considered in the analysis of these systems.

\section{Conclusion}

In this paper, based on the Euler-Bernoulli and Timoshenko beam theories, size-dependent formulations are developed for micro-scaled circular rings on elastic foundation. A displacement field is proposed for circular rings and Hamilton's principle is utilized to derive the governing equations and boundary conditions of micro-rings. As a case study, the dimensionless sizedependent natural frequencies of a circular microring with rectangular cross-section are obtained and compared with those evaluated based on the classical theory. The response of the micro-ring under application of static and harmonic point loads is investigated, and the findings are compared with classical ones. Results reveal that the normalized natural frequency of the micro ring and the deflection of micro ring under static and harmonic forces are size-dependent,

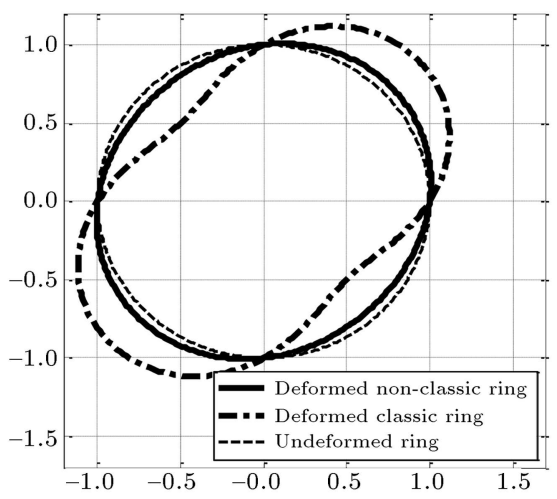

(a) $h / l=3$

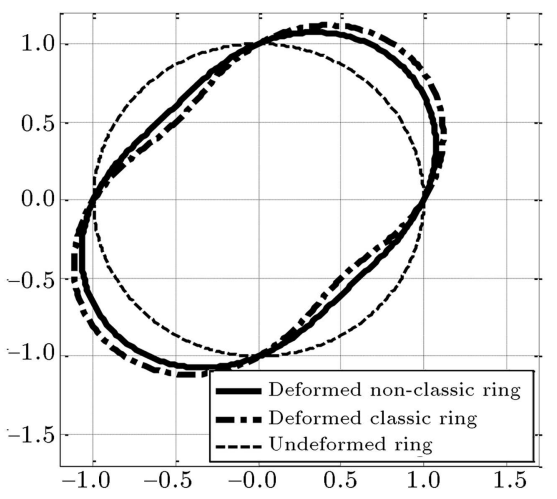

(b) $h / l=10$

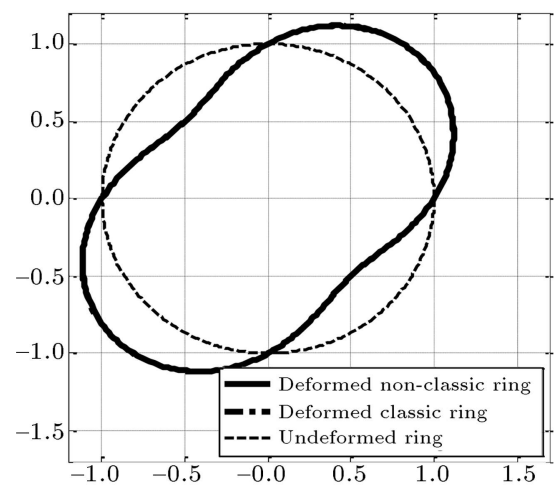

(c) $h / l>100$

Figure 10. Maximum deflection of micro-ring under harmonic load. 
while according to the classical theory, the normalized behavior of a structure is independent of its size. As the ring thickness increases, the results of the modified couple stress theory converge to those of the classical theory. Furthermore, as the radius of the ring increases, the natural frequency given by the Timoshenko beam theory converges to that predicted by the Euler-Bernoulli beam theory.

\section{References}

1. Sheng, W. and Wei, X. "A new calculation of potential energy of supporting springs and the application in design of vibrating ring gyroscope", Aerosp. Sci. Technol., 15(5), pp. 409-415 (2011).

2. Yoon, S.W., Lee, S. and Najafi, K. "Vibration sensitivity analysis of MEMS vibratory ring gyroscopes", Sensor. Actuat. A-Phys., 171(2), pp. 163-177 (2011).

3. Hu, Z.X., Gallacher, B.J., Burdess, J.S., et al. "A parametrically amplified MEMS rate gyroscope", Sensor. Actuat. A-Phys., 167(2), pp. 249-260 (2011).

4. Yeh, C.N., Tsai, J.J., Shieh, R.J., et al. "A vertically supported ring-type mems gyroscope utilizing electromagnetic actuation and sensing", in IEEE. C. Elec. Devices (2008).

5. Chang, C.-O., Chang, G.E., Chou, C.S., et al. "Inplane free vibration of a single-crystal silicon ring", Int. J. Solids. Struct., 45(24), pp. 6114-6132 (2008).

6. Wu, X. and Parker, R.G. "Vibration of rings on a general elastic foundation", J. Sound. Vib., 295(1), pp. 194-213 (2006).

7. Cooley, C.G. and Parker, R.G. "Vibration of highspeed rotating rings coupled to space-fixed stiffnesses", J. Sound. Vib., 333(12), pp. 2631-2648 (2014).

8. Patel, B.P., Ganapathi, M., Makhecha, D.P., et al. "Large amplitude free flexural vibration of rings using finite element approach", Int. J. Nonlin Mech., 38(6), pp. 911-921 (2003).

9. Fleck, N.A., Muller, G.M., Ashby, M.F., et al. "Strain gradient plasticity: theory and experiment", Acta. Metal. Mater., 42(2), pp. 475-487 (1994).

10. McFarland, A.W. and Colton, J.S. "Role of material microstructure in plate stiffness with relevance to microcantilever sensors", J. Micromech. Microeng., 15(5), p. 1060 (2005).

11. Stölken, J.S. and Evans, A.G. "A microbend test method for measuring the plasticity length scale", Acta. Mater., 46(14), pp. 5109-5115 (1998).

12. Ghayesh, M.H., Amabili, M. and Farokhi, H. "Threedimensional nonlinear size-dependent behaviour of Timoshenko microbeams", Int. J. Eng. Sci., 71, pp. 1-14 (2013).

13. Tsiatas, G.C. and Katsikadelis, J.T. "A new microstructure-dependent Saint-Venant torsion model based on a modified couple stress theory", Eur. J. Mech. A-Solid., 30(5), pp. 741-747 (2011).
14. Mindlin, R.D. and Tiersten, H.F. "Effects of couplestresses in linear elasticity", Arch. Ration. Mech. An., 11(1), pp. 415-448 (1962).

15. Asghari, M., Kahrobaiyan, M.H., Rahaeifard, M., et al. "Investigation of the size effects in Timoshenko beams based on the couple stress theory", Arch. Appl. Mech., 81(7), pp. 863-874 (2011).

16. Zeighampour, H. and Beni, Y.T. "A shear deformable cylindrical shell model based on couple stress theory", Arch. Appl. Mech., 85(4), pp. 1-15 (2014).

17. Yang, F., Chong, A.C.M., Lam, D.C.C., et al. "Couple stress based strain gradient theory for elasticity", Int. J. Solids. Struct., 39(10), pp. 2731-2743 (2002).

18. Farokhi, H., Ghayesh, M.H. and Amabili, M. "Nonlinear dynamics of a geometrically imperfect microbeam based on the modified couple stress theory", Int. J. Eng. Sci., 68, pp. 11-23 (2013).

19. Aghazadeh, R., Cigeroglu, E. and Dag, S. "Static and free vibration analyses of small-scale functionally graded beams possessing a variable length scale parameter using different beam theories", Eur. J. Mech. A-Solid., 46, pp. 1-11 (2014).

20. Rezazadeh, G., Vahdat, A.S., Tayefeh-rezaei, S., et al., "Thermoelastic damping in a micro-beam resonator using modified couple stress theory", Acta. Mech., 223(6), pp. 1137-1152 (2012).

21. Abbasnejad, B., Rezazadeh, G. and Shabani, R. "Stability analysis of a capacitive fgm micro-beam using modified couple stress theory", Acta. Mech. Solida. Sin., 26(4), pp. 427-440 (2013).

22. Şimḑek, M. "Dynamic analysis of an embedded microbeam carrying a moving microparticle based on the modified couple stress theory", Int. J. Eng. Sci., 48(12), pp. 1721-1732 (2010).

23. Rokni, H., Milani, A.S. and Seethaler, R.J. "Sizedependent vibration behavior of functionally graded CNT-Reinforced polymer microcantilevers: Modeling and optimization", Eur. J. Mech. A-Solid., 49, pp. 2634 (2015).

24. Ma, H.M., Gao, X.L. and Reddy, J.N. "A non-classical Mindlin plate model based on a modified couple stress theory", Acta. Mech., 220(1-4), pp. 217-235 (2011).

25. Zhang, B., He, D. Liu, Z. Gan, et al. "A non-classical Mindlin plate finite element based on a modified couple stress theory", Eur. J. Mech. A-Solid., 42, pp. 63-80 (2013).

26. Asghari, M., Ahmadian, M.T., Kahrobaiyan, M.H., et al. "On the size-dependent behavior of functionally graded micro-beams", Mater. Design., 31(5), pp. 23242329 (2010).

27. Asghari, M., Rahaeifard, M., Kahrobaiyan, M.H., et al. "The modified couple stress functionally graded Timoshenko beam formulation", Mater. Design., 32(3), pp. 1435-1443 (2011).

28. Chen, W.J. and Li, X.P. "Size-dependent free vibration analysis of composite laminated Timoshenko beam 
based on new modified couple stress theory", Arch. Appl. Mech., 83(3), pp. 431-444 (2013).

29. Fakhrabadi, M.M.S., Rastgoo, A., Ahmadian, M.T., et al. "Dynamic analysis of carbon nanotubes under electrostatic actuation using modified couple stress theory", Acta. Mech., 225(6), pp. 1523-1535 (2014).

30. Tsiatas, G.C. "A new Kirchhoff plate model based on a modified couple stress theory", Int. J. Solids. Struct., 46(13), pp. 2757-2764 (2009).

31. Chen, W., Xu, M. and Li, L. "A model of composite laminated Reddy plate based on new modified couple stress theory", Compos. Struct., 94(7), pp. 2143-2156 (2012).

32. Chen, W. and Li, X. "A new modified couple stress theory for anisotropic elasticity and microscale laminated Kirchhoff plate model", Arch. Appl. Mech., 84(3), pp. 323-341 (2014).

33. Gao, X.L., Huang, J.X. and Reddy, J.N. "A nonclassical third-order shear deformation plate model based on a modified couple stress theory", Acta. Mech., 224(11), pp. 2699-2718 (2013).

34. Wang, L. "Size-dependent vibration characteristics of fluid-conveying microtubes", J. Fluid. Struct., 26(4), pp. 675-684 (2010).

35. Kahrobaiyan, M.H., Asghari, M., Rahaeifard, M., et al. "Investigation of the size-dependent dynamic characteristics of atomic force microscope microcantilevers based on the modified couple stress theory", Int. J. Eng. Sci., 48(12), pp. 1985-1994 (2010).

36. Rahaeifard, M., Kahrobaiyan, M.H., Asghari, M., et al. "Static pull-in analysis of microcantilevers based on the modified couple stress theory", Sensor. Actuat. A-Phys., 171(2), pp. 370-374 (2011).

37. Ashoori, A. and Mahmoodi, M.J. "The modified version of strain gradient and couple stress theories in general curvilinear coordinates", Eur. J. Mech. ASolid., 49, pp. 441-454 (2015).

38. Rao, S.S., Vibration of Continuous Systems, New York: John Wiley \& Sons (2007).

\section{Biographies}

Ali Karimzadeh was born in 1988. He received the BS degree in Mechanical Engineering from the University of Shiraz, Iran, in 2010, and the MS degree in Mechanical Engineering from the Sharif University of Technology in 2012. Now he is a PhD candidate in the School of Mechanical Engineering in Sharif University of Technology. His research interests are in the areas of micro electromechanical systems, strength of material, and advanced finite-element method.

Mohammd Taghi Ahmadian received a $\mathrm{PhD}$ degree from the Department of Mechanical Engineering at the University of Kansas, USA, in 1986. From 1984 to 1985, he was an Assistant Professor at the University of Missouri, and from 1985 to 1988, he was an Assistant Professor in the Department of Mechanical Engineering at the University of Kansas at Lawrence. He started working as an Assistant Professor at Sharif University of Technology in 1989; at present, he is a Professor in the School of Mechanical Engineering at Sharif University of Technology, Tehran, Iran. His research interests include cellular biomechanics, soft tissue modeling, strength of material, and advanced finite-element method.

Masoud Rahaeifard was born in 1985. He received BS degree in Mechanical Engineering from the Amirkabir University of Technology, Iran, in 2007, and MS degree in Mechanical Engineering from the Sharif University of Technology in 2009. He received a PhD degree from the Department of Mechanical Engineering at the Sharif University of Technology in 2013. He has started working as an Assistant Professor at Golpayegan University of Technology from 2013. His research interests are in the areas of micro electromechanical systems, nonclassical continuum theories. 